\title{
MicroRNA Expression Profiling of Pancreatic Cancer Cell Line L3.6p1 Following B7-H4 Knockdown
}

\author{
Yun Qian ${ }^{a}$ Limin Feng ${ }^{b}$ Weigen Wu ${ }^{b}$ Tianhao Weng ${ }^{b}$ Chenyu Hu ${ }^{b}$ Bo Hong \\ Frederick X.C. Wang ${ }^{d}$ Lingwei Shen $^{\mathrm{a}}$ Qi Wang ${ }^{\mathrm{a}}$ Xin Jine Hangping Yao ${ }^{\mathrm{b}}$
}

aDepartment of Clinical Laboratory, The Second Affiliated Hospital, Zhejiang University School of Medicine, Hangzhou, 'bState Key Laboratory for Diagnosis and Treatment of Infectious Diseases, Collaborative Innovation Center for Diagnosis and Treatment of Infectious Diseases, The First Affiliated Hospital, Zhejiang University School of Medicine, Hangzhou, 'Department of Pathology, The Second Affiliated Hospital, Zhejiang University School of Medicine, Hangzhou, China; ${ }^{d}$ Department of Bioengineering, Erik Jonsson School of Engineering and Computer Science, The University of Texas at Dallas, Texas, USA; ${ }^{e}$ Department of Basic Medicine, Medical College, Shaoxing University, Shaoxing, China

\section{Key Words}

B7-H4 • MicroRNA • Profiling • Pancreatic cancer • Tumor progression

\begin{abstract}
Background/Aims: Co-stimulating molecule $\mathrm{B} 7-\mathrm{H} 4$ regulates $\mathrm{T}$ cell-mediated immune responses, participates in tumor immune escape, and promotes the proliferation and metastasis of pancreatic cancer cells. However, the specific mechanisms are unclear. MicroRNAs (miRNAs) participated in the pathogenesis and progression of cancer. Methods: In this study, a microarray technique was used to screen B7-H4-related differentially expressed miRNAs in a pancreatic cancer cell line find those associated with pancreatic cancer. Using a miRCURY'M LNA Array approach, we compared the miRNA expression profiles of L3.6p1 pancreatic cancer cells transfected with B7-H4 siRNA for $72 \mathrm{~h}$ with those transfected with non-target siRNAs. Results: B7-H4 siRNA significantly up-regulated 57 miRNAs and down-regulated 14 miRNAs. Gene Ontology (GO) and Kyoto Encyclopedia of Genes and Genomes (KEGG) Pathway analysis of predicted miRNA targets showed that these genes were mainly involved in protein binding, pathways in cancer, mitogen-activated protein kinase (MAPK) signaling pathway, and phosphatidylinositol 3-kinase-Akt (PI3K-Akt) signaling pathway. Conclusions: This is the first description of target genes of B7-H4, showing that miRNAs participate in the B7-H4 mediated regulation of oncogenicity and pathogenesis of pancreatic cancer. These results may help us better understand the role of $\mathrm{B} 7-\mathrm{H} 4$ in the progression of pancreatic cancer and its possible mechanisms. We also provide novel biomarkers for potential treatments of pancreatic cancer.

Y. Qian and L. Feng contributed equally to this article.




\section{Cellular Physiology Cell Physiol Biochem 2017;44:494-504 and BiOChemistry Published online: November 17, 2017 \begin{tabular}{l|l} 
DOI: 10.1159/000485086 2017 The Author(s). Published by S. Karger AG, Basel \\
www.karger.com/cpb
\end{tabular} \\ Qian et al.: B7-H4-Associated MicroRNA Expression Profiling}

\section{Introduction}

Pancreatic cancer is one of the most common types of tumors in humans [1]. Many researches have shown that the initiation and development of pancreatic cancer are closely related to the tumor microenvironment. Specifically, B7/CD28 co-signaling molecules play a crucial role [2, 3]. B7-H4 (also named B7S1 or B7x), a member of the B7 superfamily [4, 5], has been suggested to play an important role in tumor progression and immune escape. In our previous study, we found that B7-H4 was associated with poor prognosis in patients with pancreatic cancer and it promoted tumor progression through multiple processes, including the inhibition of apoptosis and induction of the extracellular regulated protein kinases $1 / 2$ (Erk1/2) signaling pathway [6-8]. However, the exact mechanism of action for B7-H4 this cancer biology remains unclear. Thus, studying the role of B7-H4 in tumor progression is essential for developing more effective treatments and improving patients' prognoses for pancreatic cancer.

Recently, novel gene expression-related mechanisms underpinning tumor initiation have been identified. miRNAs are 20-22 nucleotide long endogenous noncoding RNAs that repress target gene expression at the post-transcriptional level, which are now considered to be master regulators of gene expression for at least 30\% of human genes [9]. Functional studies indicate that miRNAs have important functions in tumor occurrence and development $[10,11]$. Recent studies have revealed that miRNAs are associated with the risk of different cancers, such as lung cancer [12], colorectal cancer [13], bladder cancer [14], breast cancer [15], and pancreatic cancer [16, 17]. All of these researches have provided strong evidence that miRNAs play a key role during tumorigenesis and tumor progression.

Given the significant role of miRNAs in gene regulation and tumorigenesis, miRNAs might interact with the B7 superfamily in tumors. For instance, IFN-gamma induces B7-H1 protein expression and alters the miRNA expression profiles in cholangiocytes [18]. miR152 directly bind to B7-H1 3' untranslated region and inhibits B7-H1 expression, which enhances T cell proliferation and effector cytokine production via inhibition of the B7-H1/ PD-1 pathway [19]. Furthermore, over-expression of B7-H4 promoted tumor growth through TGF- $\beta 1$, which increased expression of miR-155 through SMAD3 and SMAD4 and attenuated miR-143 through CEBPB [20].

Thus, we hypothesized that miRNAs regulated by B7-H4 might influence pancreatic cancer risk. So far, there are no reports about the miRNA expression profile following B7$\mathrm{H} 4$ induction in a pancreatic cancer cell model. In this study, we profiled the miRNAs of the L3.6p1 pancreatic cancer cell line infected with B7-H4 small interfering RNA (siRNA), and 71 miRNAs were found to be differentially expressed. Some of the differentially expressed miRNAs might be potential therapeutic targets for pancreatic cancer. Thus, we provide a new context in which to study the mechanism by which B7-H4 promotes tumor progression.

\section{Materials and Methods}

\section{Cells and infection}

L3.6p1 cells, which were derived from a human pancreatic carcinoma, were grown and infected with B7-H4 siRNA as previously described. The siRNA sequences are listed in Table 1, and the efficiency of inhibition of B7-H4 mRNA expression was found to be up to 78.7\% [8].

\section{MiRNA microarray analysis}

For the total RNA extraction, L3.6p1 cells infected with $B 7-H 4$ siRNA for $72 \mathrm{~h}$ were harvested. Total RNA was extracted using TRIzol (Invitrogen, CA, USA) and an miRNeasy Mini Kit (Qiagen, MD, USA) according to the manufacturer's instructions. The concentration of RNA was determined using a Nanodrop ND-1000 Spectrophotometer (Thermo Scientific, MA, USA). The samples were then labeled using the miRCURY'm $\mathrm{Hy}^{\mathrm{TM}} / \mathrm{Hy}^{\mathrm{TM}}$ Power Labeling Kit (Exiqon, MA, USA) and hybridized on the miRCURY' ${ }^{\mathrm{TM}}$ LNA MicroRNA Arrays (v.16.0, Exiqon). Following the washing steps, the slides were scanned using the Axon GenePix 4000B microarray scanner (Axon Instruments, CA, USA). 


\section{Cellular Physiology Cell Physiol Biochem 2017;44:494-504 \begin{tabular}{l|l|l}
\hline and & DOI: 10.1159/000485086 & $\begin{array}{l}\text { C } 2017 \text { The Author(s). Published by S. Karger AG, Basel } \\
\text { www.karger.com/cpb }\end{array}$
\end{tabular}

Table 1. siRNA oligonucleotides specific for B7-H4

\begin{tabular}{lcc}
\hline siRNA & & Oligonucleotide \\
\hline B7-H4 siRNA & target & 5'-GGG AGA CAC TCC ATC ACA GTC ACT A-3' (97-121) \\
& sense strand & 5'-GGG AGA CAC UCC AUC ACA GUC ACU A (dTdT)-3' \\
& antisense strand & 5'-UAG UGA CUG UGA UGG AGU GUC UCC C(dTdT)-3' \\
& \\
\hline
\end{tabular}

Scanned images were imported into GenePix Pro 6.0 software (Axon Instruments) for grid alignment and data extraction. Replicated miRNAs were averaged and miRNAs with intensities $\geq 50$ in all samples were chosen to calculate the normalization factor. Expressed data were normalized using the median normalization. After normalization, significantly differentially expressed miRNAs were identified using Volcano Plot filtering. Finally, hierarchical clustering was performed to identify distinguishable miRNA expression profiling among the samples.

The differential expression of miRNAs between groups was assessed by one-way analysis of variance (ANOVA), and the correction of Benjamini-Hochberg FDR was applied, in addition to a Tukey's honestly significant difference (HSD) post-hoc test. The expression change (fold-change) of a miRNA in a $B 7-H 4$ siRNA-infected sample relative to the non-target siRNA-infected sample was calculated. Significance was determined using a fold-change threshold of at least 2 or at most 0.5 , and a $P$ value cut-off of 0.05 was applied. A fold-change of 2 or 0.5 was chosen to improve the accuracy of the results.

\section{Bioinformatics analysis}

The miRNA targets were predicted using the TargetScan version 7.1 database and miRanda version 3.3a database, which were downloaded from http://www.targetscan.org/vert_71/ and http://www. microrna.org/microrna/home.do/, respectively. To more strictly predict targets, the predicted miRNA targets found for the differentially expressed miRNAs in the TargetScan version 7.1 database were selected using the cutoff of a context score percentile $>50$. In the miRanda version 3.3a database, these were selected using the Max Energy <-10. Ultimately, the final target genes for the miRNAs were selected from the overlap of the gene lists predicted using the two software programs. The predicted targets underwent GO (http:// www.geneontology.org/) and KEGG (http://www.genome.jp/kegg/) pathway analyses using the functional annotation tools of the Database from Annotation, Visualization, and Integrated Discovery (DAVID; http:// david.abcc.Ncifcrf.gov/). The enrichment of GO terms and KEGG pathways were selected using a cutoff standard of $P<0.01$ and $\mathrm{FDR}<1$.

\section{Statistical analysis}

The results were reported as the means \pm SD for triplicate measurements. Statistically significant differences between groups were estimated by the Student's $t$ test using SPSS software (ver. 13.0). Differences of $P<0.05$ were considered statistically significant. All the analyses using DAVID involved the Fisher Exact test. When this test yielded $P<0.05$, the relevant data were considered statistically significant.

\section{Results}

\section{MiRNA expression profiles following B7-H4 inhibition}

To understand the effect of B7-H4 inhibition on miRNAs in L3.6p1 pancreatic cancer cells, we profiled the expression of cellular miRNAs in response to B7-H4 siRNA infection. Among the 938 miRNAs on the arrays, 255 were detected in L3.6p1 cells which then were involved in a comparative analysis of miRNA expression patterns. The miRNA expression pattern induced after exposure to $\mathrm{B} 7-\mathrm{H} 4$ siRNA differed from that of the cells treated with non-target siRNA. Therefore, B7-H4 inhibition altered discrete miRNA expression patterns. 


\section{Cellular Physiology Cell Physiol Biochem 2017;44:494-504

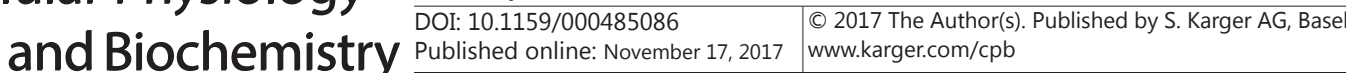

After B7-H4 inhibition, 71 miRNAs exhibited differential expression. Among these, 57 $(80 \%)$ were up-regulated, while $14(20 \%)$ were down-regulated (Fig. 1). The fold-change of miRNAs after $B 7-H 4$ siRNA transfection is shown in Table 2.

GO analysis of predicted target genes of differentially expressed miRNAs

To determine the roles of differentially expressed miRNAs in response to B7-H4 inhibition, potential target genes of these miRNAs were predicted using miRanda version 3.3a with a Max Energy $<-10$ and TargetScan version 7.1 with a context score percentile > 90.

Following B7-H4 expression inhibition, 16, 150 predicted targets were obtained for the 71 differentially expressed miRNAs. The 16, 150 predicted target genes were then subjected to $\mathrm{GO}$ analysis in DAVID v6.7. With the cutoff standard of $P<0.01$ and FDR $<1,227$ discrete cohorts of GO terms for molecular function were detected to be enriched following B7-H4 inhibition. Among these, the top 10 GO terms were protein binding, cytoplasm, membrane, regulation of transcription, DNA-template and sequence-specific DNA binding transcription factor activity, ATP binding, cytosol, transcription, nucleoplasm, and metal ion binding (Fig. 2). Interestingly, these results indicated that B7-H4 might act as a factor controlling general levels of gene expression.

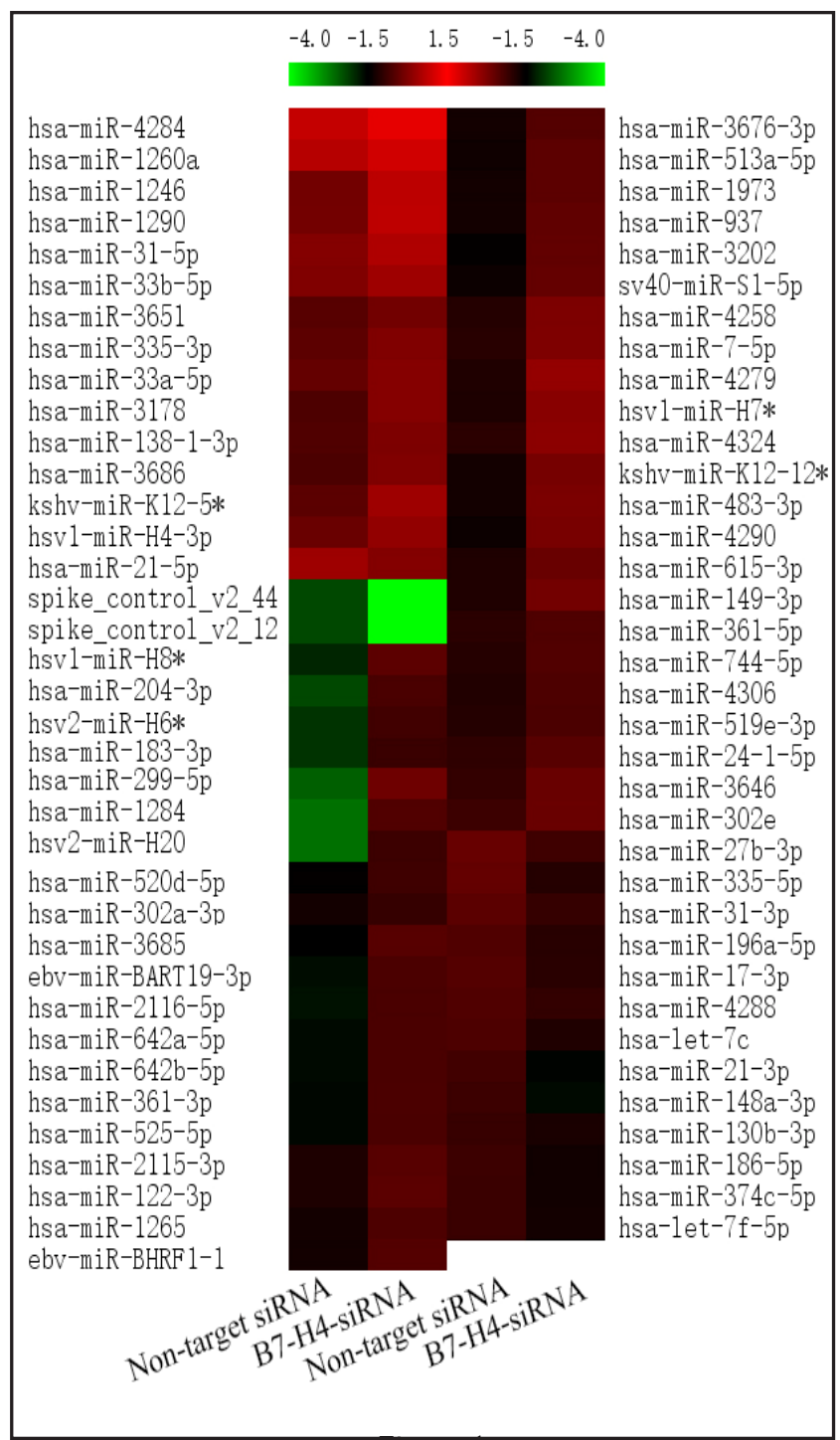

Fig. 1. Distinct changes in miRNA expression following B7-H4 knockdown. The columns correspond to expression patterns for differentially expressed miRNAs after infection with B7H4 siRNA for 72 h. Significance was determined using a foldchange threshold of at least 2 or at most 0.5 and a P-value cutoff of 0.05 . The red color represents up-regulation, while the green color indicates down-regulation.

\section{KEGG pathway analysis of predicted target genes of differentially expressed miRNAs}

To better understand the effect of the differentially expressed miRNAs following B7-H4 expression inhibition, their predicted targets were conducted with KEGG pathway analysis using DAVID 6.7. We found that 130 pathways were significantly enriched. The top 10 were pathways related to cancer, the PI3K-Akt signaling pathway, the Rap1 signaling pathway, endocytosis, the MAPK signaling pathway, the Ras signaling pathway, axon guidance, the Hippo signaling pathway, the thyroid hormone signaling pathway, and proteoglycans in cancer (Fig. 3). The network for these miRNAs and target genes is shown in Fig. 4A. Notably, 
Table 2. Fold-changes of differentially expressed miRNAs in B7-H4 siRNA-infected cells. Fold-change: B7H4 siRNA vs. non-target siRNA

\begin{tabular}{|c|c|c|c|c|c|}
\hline ID & Name & Fold change & ID & Name & Fold change \\
\hline 148633 & hsa-miR-299-5p & 174.43 & 146161 & hsa-miR-2115-3p & 4.77 \\
\hline 46336 & hsa-miR-1284 & 119.19 & 46737 & hsa-miR-1265 & 4.69 \\
\hline 147738 & hsv2-miR-H20 & 65.84 & 147595 & hsa-miR-3178 & 4.42 \\
\hline 42502 & hsa-miR-204-3p & 39.05 & 148481 & hsa-miR-3646 & 4.24 \\
\hline 146042 & hsv1-miR-H8* & 29.72 & 148156 & hsa-miR-3686 & 4.05 \\
\hline 147767 & hsa-miR-4279 & 22.63 & 45764 & hsa-miR-302e & 3.41 \\
\hline 147900 & hsv2-miR-H6* & 19.44 & 42872 & hsa-miR-138-1-3p & 3.36 \\
\hline 146090 & hsv1-miR-H7* & 17.99 & 11052 & hsa-miR-31-5p & 3.29 \\
\hline 147920 & hsa-miR-4290 & 17.59 & 147722 & hsa-miR-4306 & 3.26 \\
\hline 148682 & hsa-miR-483-3p & 15.99 & 27568 & hsa-miR-744-5p & 3.21 \\
\hline 17953 & hsa-miR-183-3p & 15.75 & 146159 & hsv1-miR-H4-3p & 3.04 \\
\hline 42516 & kshv-miR-K12-12* & 14.21 & 146043 & hsa-miR-24-1-5p & 3.03 \\
\hline 147847 & hsa-miR-4324 & 13.51 & 11164 & hsa-miR-519e-3p & 2.94 \\
\hline 146158 & hsa-miR-3202 & 11.71 & 146113 & hsa-miRPlus-G1246-3p & 2.79 \\
\hline 42522 & ebv-miR-BART19-3p & 11.60 & 14301 & hsa-miR-361-5p & 2.64 \\
\hline 146010 & hsa-miR-2116-5p & 11.28 & 145745 & hsa-miR-335-3p & 2.55 \\
\hline 147631 & hsa-miR-4258 & 11.05 & 147636 & hsa-miR-4284 & 2.51 \\
\hline 148032 & hsa-miR-3685 & 10.47 & 147203 & hsa-miR-302a-3p & 2.41 \\
\hline 145865 & hsa-miR-361-3p & 10.05 & 145859 & hsa-miR-33a-5p & 2.41 \\
\hline 6 & hsa-miR-642a-5p/ & 100 & 145950 & hsa-miR-33b-5p & 2.30 \\
\hline 42007 & hsa-miR-642b-5p & 10.05 & 148327 & hsa-miR-3651 & 2.08 \\
\hline 29490 & hsa-miR-7-5p & 9.96 & 145975 & hsa-miR-1260a & 2.06 \\
\hline 17492 & sv40-miR-S1-5p & 9.95 & 147512 & hsa-miR-21-5p & 0.49 \\
\hline 42486 & hsa-miR-149-3p & 9.76 & 10936 & hsa-miR-130b-3p & 0.47 \\
\hline 11175 & hsa-miR-525-5p & 9.73 & 147588 & hsa-miR-4288 & 0.46 \\
\hline 42581 & hsa-miR-513a-5p & 8.17 & 17752 & hsa-let-7f-5p & 0.38 \\
\hline 42514 & hsa-miR-937 & 7.57 & 46320 & hsa-miR-31-3p & 0.37 \\
\hline 46921 & hsa-miR-1290 & 7.57 & 10990 & hsa-miR-196a-5p & 0.35 \\
\hline 148642 & hsa-miR-1246 & 7.28 & 148430 & hsa-miR-374c-5p & 0.35 \\
\hline 27672 & hsa-miR-615-3p & 7.20 & 147199 & hsa-miR-27b-3p & 0.34 \\
\hline 146165 & hsa-miR-1973 & 7.14 & 19588 & hsa-miR-17-3p & 0.32 \\
\hline 147885 & kshv-miR-K12-5* & 6.18 & 18739 & hsa-miR-186-5p & 0.31 \\
\hline 148410 & hsa-miR-3676-3p & 5.70 & 145820 & hsa-let-7c & 0.29 \\
\hline 42906 & ebv-miR-BHRF1-1 & 5.65 & 11065 & hsa-miR-335-5p & 0.19 \\
\hline 42551 & hsa-miR-122-3p & 5.23 & 10955 & hsa-miR-148a-3p & 0.15 \\
\hline 46354 & hsa-miR-520d-5p & 5.13 & 42524 & hsa-miR-21-3p & 0.14 \\
\hline
\end{tabular}

enrichment for the above pathways suggested that B7-H4-related miRNAs may significantly affect pathways involved in tumorigenesis.

Further, we focused on the top 10 up-regulated and top 10 down-regulated miRNAs and their targets which might have key regulation roles in B7-H4 related cancer development according to the published data. And we mapped the network of miRNAs-target gene and potential mechanism prediction in Fig. 4B, in order to establish foundation to reveal the potential mechanism of B7-H4 promoting pancreatic cancer through miRNA. Fig. 4B showed miRNAs and their target genes in initiation, growth and metastasis of tumor. The chart displayed the differentially expressed miRNAs and their target genes that were involved in tumor progression. This also included some identified upstream molecules which regulated their expression. Downregulation or upregulation of a specific miRNA was represented by a downward (red) or an upward (black) arrow, respectively. The changes of target genes expression levels inversely correlated with that of the miRNAs, and were similarly represented by an up (black) or down (red) arrow. All listed targets have been validated according to the published data. 
Fig. 2. Significantly enriched GO terms for molecular function for the predicted targets. The significantly enriched GO terms for molecular function for the predicted targets of the differentially expressed miRNAs following B7-H4 expression inhibition.

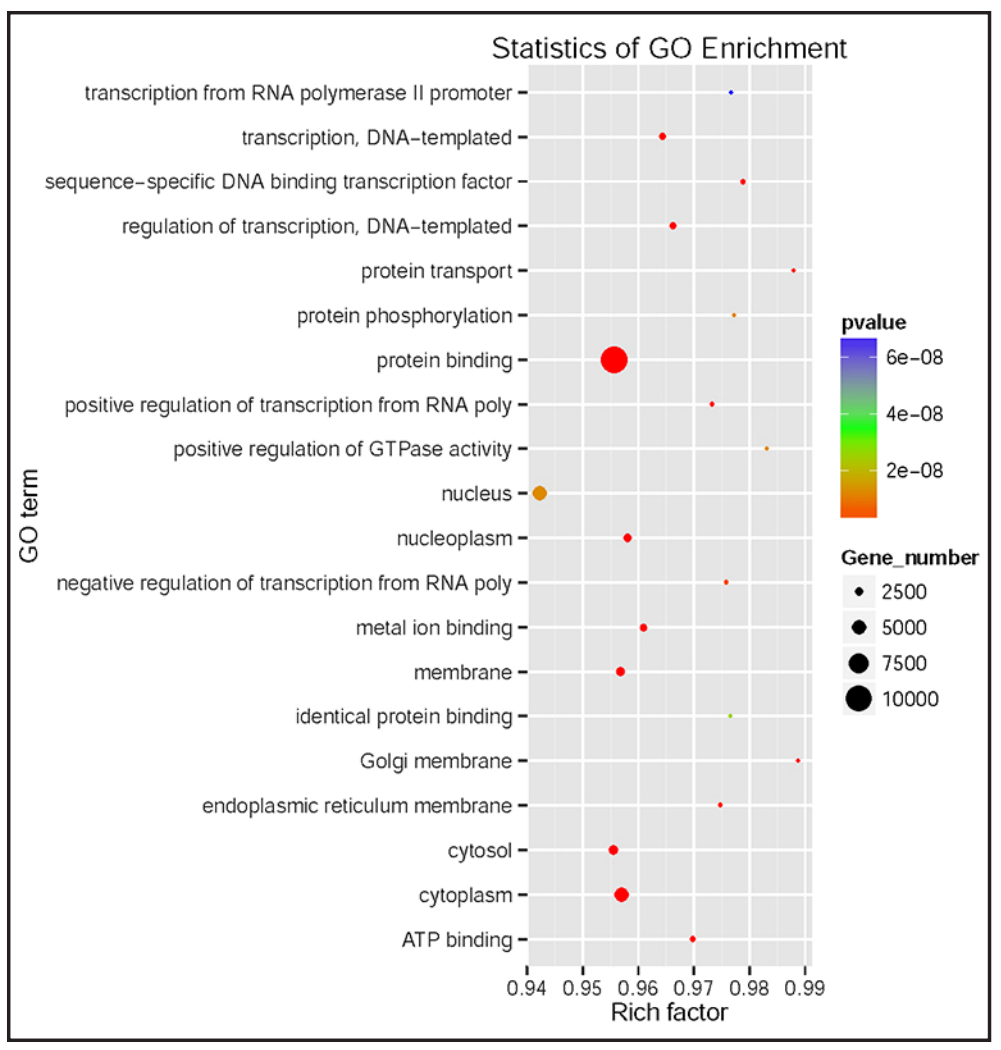

Fig. 3. KEGG pathway analysis for the predicted targets of the differentially expressed miRNAs. The significantly enriched pathways for the predicted targets of the differentially expressed miRNAs in response to B7-H4 expression inhibition.

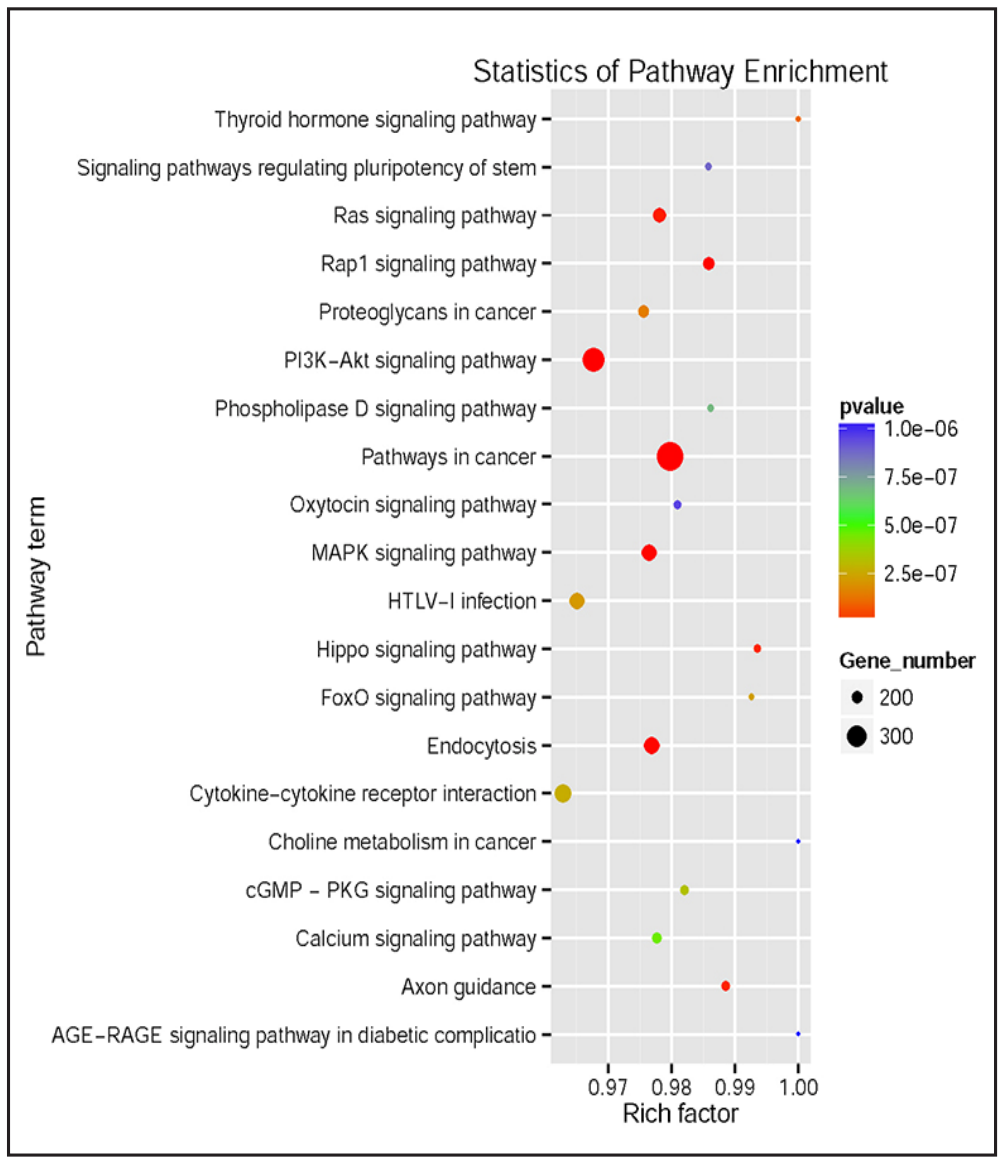




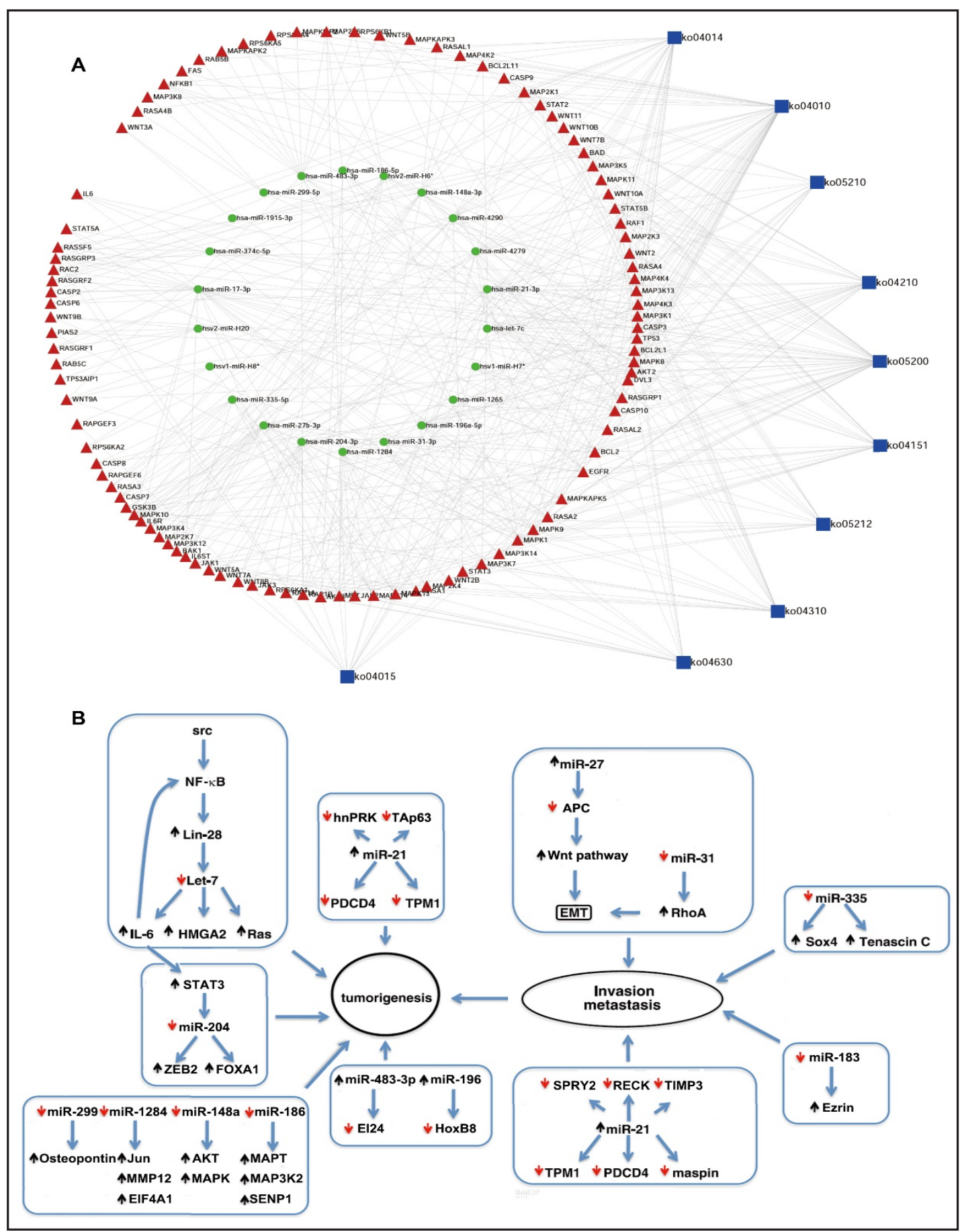

Fig. 4. Network of miRNAs and target genes. A: The network of top fold-change miRNAs (green nodes) and their target genes (red nodes). The pathways are indicated as blue nodes. B: The network of miRNAs-target gene and potential mechanism prediction. Upregulation or downregulation of a specific miRNA or target gene is represented by an upward (black) or a downward (red) arrow, respectively.

\section{Discussion}

Recently, many studies have shown that miRNAs are involved in various diseases, including cancers [10-17]. Using high-throughput RNA sequencing and bioinformatics, an increasing number of functional miRNAs have been discovered, which might participate in KARGER 


\section{Cellular Physiology Cell Physiol Biochem 2017;44:494-504

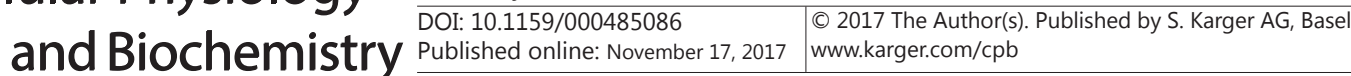 \\ Qian et al.: B7-H4-Associated MicroRNA Expression Profiling}

tumor development and could provide novel targets for pancreatic cancer therapies [21, 22]. However, the mechanisms by which miRNAs contribute to cancer progression still remain unclear.

We have studied the expression of B7-H4 in a variety of human pancreatic cancer cells and the effects of B7-H4 in tumor development. It was confirmed that B7-H4 was highly expressed in different pancreatic cancer cells, especially in L3.6p1 pancreatic cancer cells [8]. At the same time, L3.6p1 cells showed a high degree of aggressive growth in vitro [23], and B7-H4 was involved in the migration of L3.6p1 cells [8]. Therefore, we selected L3.6p1 cells as the object. In this study, we profiled miRNA expression patterns in L3.6p1 pancreatic cancer cells and found that 57 miRNAs were up-regulated and 14 were downregulated in response to B7-H4 knockdown. Many of these miRNAs have known functions and are involved in cancer biology. Notably, $m i R-299-5 p$ and $m i R-1284$ were significantly upregulated, while $m i R-21$ and $m i R-335-5 p$ was significantly down-regulated following B7-H4 knockdown according to our microarray. These miRNAs are involved in the development of tumors [24-26]. Furthermore, hsa-mir-299-5p, which targets osteopontin, plays a critical role in enhancing proliferation, tumorigenicity, and the ability of spheroid-forming breast cancer cells to display vasculogenic mimicry [27]. Additionally, miR-1284 overexpression might act as a suppressor of gastric cancer by controlling cell proliferation and apoptosis [28]. One study demonstrated that miR-1284 could inhibit cell viability via regulation of the expression of $p 27$ and induce apoptosis via regulation of the PI3K/Akt pathway in ovarian cancer cells [25]. miR-21 could decrease Fox01 protein levels, resulting in a significant increase in pancreatic ductal adenocarcinoma growth, which may act as a novel therapeutic target for inhibiting the growth of pancreatic cancer [29]. Furthermore, miR-335-5p is an important miRNA that has multiple functions in cancer biology. Ectopic expression of $m i R$ 335-5p in pancreatic cancer cell lines significantly suppresses cell growth by inhibiting c-met [30]. Thus, it is reasonable to hypothesize that $m i R-299-5 p, m i R-1284, m i R-21$, and $m i R-335$ $5 p$ might take an important part in response to B7-H4 knockdown in pancreatic cancer cells. However, this assumption needed further confirmation in future research.

As far as we know, this was the first time that the expression of miRNAs has been shown to be regulated by B7-H4. Because miRNAs play a crucial role in tumorigenesis by regulating cell proliferation and apoptosis, our study provides and new and exciting context in which to investigate B7-H4 function in tumors. A great deal of evidence indicated that B7-H4 might activate or repress some transcription factors. For instance, B7-H4 facilitates proliferation, invasion, and migration of esophageal squamous cell carcinoma cells [31] and colorectal carcinoma cells [32] by promoting a positive feedback loop involving interleukin-6 / janus kinase 2/ signal transducers and activators of transcription 3 (IL-6/JAK2/STAT3) pathway activation. In contrast, B7-H4 inhibits T-cell activation and proliferation via the inhibition of NF-kappaB nuclear translocation [33]. These transcription factors might regulate the expression of miRNAs [34, 35]. Thus, it was useful to investigate whether B7-H4 regulates the expression of miRNAs in our experimental context. We analyzed the interactions between B7-H4 and its target miRNAs with miRNA target prediction software, and found that 71 miRNAs exhibited differential expression. Furthermore, we reconstructed the network of miRNA interactions using Cytoscape software. These data suggested that B7-H4 might regulate gene expression through a miRNA-specific mechanism. The specific mechanisms by which B7-H4 promotes tumor development via regulation of miRNAs were beyond the scope of this study. However, our data suggested that several of the miRNAs observed here might be important factors mediating the pathogenesis of pancreatic cancer.

To deduce the functions of the identified miRNAs, we carried out a GO enrichment analysis according to the enriched mRNAs targeted by the significantly changed miRNAs. We found that regulation of transcription, DNA-template and sequence-specific DNA binding transcription factor activity, transcription, and DNA-template were consistent with the important roles for B7-H4 in regulating gene expression. This GO enrichment analysis suggests that B7-H4-regulated miRNAs may take part in regulating these biological processes. 
Moreover, KEGG pathway enrichment analysis identified the pathways and molecular interactions in which the differentially expressed miRNAs could be acting. Among them, cancer-related pathways, the PI3K-Akt signaling pathway, and the MAPK signaling pathway were the main pathways identified, which is consistent with our previous findings that B7$\mathrm{H} 4$ inhibition has anti-tumor effects via multiple targets, including decreasing oncogenicity, inducing apoptosis, and decreasing Erk1/2 phosphorylation [8]. These results implyed that B7-H4-related miRNAs might significantly affect these pathways to contribute to tumorigenesis [24-26]. It was well-known that the PI3K-Akt and MAPK signaling pathways are involved in the processes of tumor cell apoptosis and tumor development [36, 37]. Studies have shown that $\mathrm{B} 7-\mathrm{H} 4$ promotes lung tumor growth, progression, and metastasis through the AKT pathway [38]. In a mouse T cell model, B7-H4 inhibits T-cell proliferation and IL-2 production by inhibiting the activation of ERK, JNK, and AKT [39]. All of these reports came to the conclusion that B7-H4 is involved in the pathological process of tumorigenesis through PI3K-Akt and MAPK signaling pathway. Our pathway enrichment analysis of the miRNApredicted targets showed the PI3K-Akt and MAPK signaling pathways were enriched. Thus, some of the differentially expressed miRNAs induced by B7-H4 may affect the pathological process of tumorigenesis by modulating the PI3K-Akt and MAPK signaling pathways.

In conclusion, our study identified miRNAs under B7-H4 regulation. We constructed miRNA networks regulated by B7-H4 and took advantage of GO and KEGG analyses to show that B7-H4-regulated miRNAs likely play an important role in tumor development by affecting pathways associated with cancer, the PI3K-Akt signaling pathway, and the MAPK signaling pathway. We will investigate the function of these miRNAs and their targets in order to find the downstream signaling molecules of B7-H4 in pancreatic cancer. Thus, we can improve our understanding of B7-H4-induced oncogenicity in a future study.

\section{Abbreviations}

miRNAs (MicroRNAs); GO (Gene Ontology); KEGG (Kyoto Encyclopedia of Genes and Genomes); MAPK (mitogen-activated protein kinase); PI3K (phosphatidylinositol 3-kinase); Erk (extracellular regulated protein kinases); siRNA (small interfering RNA); ANOVA (oneway analysis of variance); DAVID (Database from Annotation, Visualization, and Integrated Discovery); IL (interleukin); STAT3 (signal transducers and activators of transcription 3); JAK2 (janus kinase2).

\section{Acknowledgements}

This work was supported by grants from the National Natural Science Foundation of China [grant number 30972777]; and the Zhejiang Provincial Natural Science Foundation of China [grant number LY15H190002 and LQ16H160005]; and the technological research project for public welfare of Shaoxing [grant number 2014B70051] of China. The funders had no role in study design, data collection and analysis, decision to publish, or preparation of the manuscript. Qian Yun, Wu Weigen and Weng Tianhao designed the cell experiments. Hu Chenyu, Hong Bo, Shen Lingwei and Wang Qi performed the miRNA microarray analysis. Wang Frederick X.C. and Feng Liming performed the statistical analysis. Yao Hangping and Jin Xin conceived and directed the project. All authors participated in writing the manuscript.

\section{Disclosure Statement}

The authors declare that they have no conflicts of interest related to the publication of this manuscript. 


\section{Cellular Physiology Cell Physiol Biochem 2017;44:494-504 \begin{tabular}{l|l|l|} 
and BOI: 10.1159/000485086 & $\begin{array}{l}\text { C) } 2017 \text { The Author(s). Published by S. Karger AG, Basel } \\
\text { www.karger.com/cpb }\end{array}$
\end{tabular} \\ Qian et al.: B7-H4-Associated MicroRNA Expression Profiling}

\section{References}

1 Stewart BW, Wild CP: World Cancer Report 2014. International Agency for Research on Cancer 2014;Lyon, France, 2014.

2 Xie C, Liu D, Chen Q, Yang C, Wang B, Wu H: Soluble B7-H3 promotes the invasion and metastasis of pancreatic carcinoma cells through the TLR4/NF-кB pathway. Sci Rep 2016;6:27528.

-3 Zhang Y, Zhang J, Xu K, Xiao Z, Sun J, Xu J, Wang J, Tang Q: PTEN/PI3K/mTOR/B7-H1 signaling pathway regulates cell progression and immuno-resistance in pancreatic cancer. Hepatogastroenterology 2013;60:1766-1772.

-4 Sica GL, Choi IH, Zhu G, Tamada K, Wang SD, Tamura H, Chapoval AI, Flies DB, Bajorath J, Chen L: B7-H4, a molecule of the B7 family, negatively regulates T cell immunity. Immunity 2003;18:849-861.

5 Prasad DV, Richards S, Mai XM, Dong C: B7S1, a novel B7 family member that negatively regulates T cell activation. Immunity 2003;18:863-873.

-6 Qian Y, Sang Y, Wang FX, Hong B, Wang Q, Zhou X, Weng T, Wu Z, Zheng M, Zhang H, Yao H: Prognostic significance of B7-H4 expression in matched primary pancreatic cancer and liver metastases. Oncotarget 2016;7:72242-72249.

7 Shen L, Qian Y, Wu W, Weng T, Wang FXC, Hong B, Wu Z, Wang Q, Sang Y, Zhang H, Zhou X, Yao H: B7-H4 is a prognostic biomarker for poor survival in patients with pancreatic cancer. Hum Pathol 2017;66:79-85.

8 Qian Y, Hong B, Shen L, Wu Z, Yao H, Zhang L: B7-H4 enhances oncogenicity and inhibits apoptosis in pancreatic cancer cells. Cell Tissue Res 2013;353:139-151.

-9 Barbato C, Arisi I, Frizzo ME, Brandi R, Da Sacco L, Masotti A: Computational challenges in miRNA target predictions: to be or not to be a true target? J Biomed Biotechnol 2009;803069.

10 Xu Z, Li C, Qu H, Li H, Gu Q, Xu J: MicroRNA-195 inhibits the proliferation and invasion of pancreatic cancer cells by targeting the fatty acid synthase/Wnt signaling pathway. Tumour Biol 2017;39:1010428317711324.

-11 Peng L, Liu Z, Xiao J, Tu Y, Wan Z, Xiong H, Li Y, Xiao W: MicroRNA-148a suppresses epithelial-mesenchymal transition and invasion of pancreatic cancer cells by targeting Wnt10 b and inhibiting the Wnt/ $\beta$-catenin signaling pathway. Oncol Rep 2017;38:301-308.

12 Qiu J, Hao Y, Huang S, Ma Y, Li X, Li D, Mao Y: MiR-557 works as a tumor suppressor in human lung cancers by negatively regulating LEF1 expression. Tumour Biol 2017;39:1010428317709467.

13 Li Y, Sun Z, Liu B, Shan Y, Zhao L, Jia L: Tumor-suppressive miR-26a and miR-26b inhibit cell aggressiveness by regulating FUT4 in colorectal cancer. Cell Death Dis 2017;8:e2892.

14 Liang Z, Wang X, Xu X, Xie B, Ji A, Meng S, Li S, Zhu Y, Wu J, Hu Z, Lin Y, Zheng X, Xie L, Liu B: MicroRNA-608 inhibits proliferation of bladder cancer via AKT/FOXO3a signaling pathway. Mol Cancer 2017;16:96.

15 Zhang G, Zhang W, Li B, Stringer-Reasor E, Chu C, Sun L, Bae S, Chen D, Wei S, Jiao K, Yang WH, Cui R, Liu R, Wang L: MicroRNA-200c and microRNA- 141 are regulated by a FOXP3-KAT2B axis and associated with tumor metastasis in breast cancer. Breast Cancer Res 2017;19:73.

16 Deng T, Yuan Y, Zhang C, Zhang C, Yao W, Wang C, Liu R, Ba Y: Identification of Circulating MiR-25 as a Potential Biomarker for Pancreatic Cancer Diagnosis. Cell Physiol Biochem 2016;39:1716-1722.

17 Sun XJ, Liu BY, Yan S, Jiang TH, Cheng HQ, Jiang HS, Cao Y, Mao AW: MicroRNA-29a Promotes Pancreatic Cancer Growth by Inhibiting Tristetraprolin. Cell Physiol Biochem 2015;37:707-718.

$>18$ Gong AY, Zhou R, Hu G, Li X, Splinter PL, O’Hara SP, LaRusso NF, Soukup GA, Dong H, Chen XM: MicroRNA-513 regulates B7-H1 translation and is involved in IFN-gamma-induced B7-H1 expression in cholangiocytes. J Immunol 2009;182:1325-1333.

19 Wang Y, Wang D, Xie G, Yin Y, Zhao E, Tao K, Li R: MicroRNA-152 regulates immune response via targeting B7-H1 in gastric carcinoma. Oncotarget 2017;8:28125-28134.

20 Zhou X, Mao Y, Zhu J, Meng F, Chen Q, Tao L, Li R, Fu F, Liu C, Hu Y, Wang W, Zhang H, Hua D, Chen W, Zhang X: TGF- $\beta 1$ promotes colorectal cancer immune escape by elevating B7-H3 and B7-H4 via the miR-155/miR143 axis. Oncotarget 2016;7:67196-67211.

21 Song B, Zheng K, Ma H, Liu A, Jing W, Shao C, Li G, Jin G: miR-429 determines poor outcome and inhibits pancreatic ductal adenocarcinoma growth by targeting TBK1. Cell Physiol Biochem 2015;35:1846-1856.

22 Song B, Zhang C, Li G, Jin G, Liu C: MiR-940 inhibited pancreatic ductal adenocarcinoma growth by targeting MyD88. Cell Physiol Biochem 2015;35:1167-1177. 


\section{Cellular Physiology Cell Physiol Biochem 2017;44:494-504 \begin{tabular}{l|l|l} 
DOI: 10.1159/000485086 & $\begin{array}{l}\text { O 2017 The Author(s). Published by S. Karger AG, Basel } \\
\text { www.karger.com/cpb }\end{array}$
\end{tabular} \\ Qian et al.: B7-H4-Associated MicroRNA Expression Profiling}

-23 Zeng JY, Sharma S, Zhou YQ, Yao HP, Hu X, Zhang R, Wang MH: Synergistic activities of MET/RON inhibitor BMS-777607 and mTOR inhibitor AZD8055 to polyploid cells derived from pancreatic cancer and cancer stem cells. Mol Cancer Ther 2014;13:37-48.

-24 Azarbarzin S, Hosseinpour Feizi MA, Safaralizadeh R, Ravanbakhsh R, Kazemzadeh M, Fateh A, Karimi N, Moaddab Y: The Value of miR-299-5p in Diagnosis and Prognosis of Intestinal-Type Gastric Adenocarcinoma. Biochem Genet 2016;54:413-420.

-25 Pan C, Wang D, Zhang Y, Yu W: MicroRNA-1284 Inhibits Cell Viability and Induces Apoptosis of Ovarian Cancer Cell Line OVCAR3. Oncol Res 2016;24:429-435.

-26 Slattery ML, Herrick JS, Mullany LE, Valeri N, Stevens J, Caan BJ, Samowitz W, Wolff RK: An evaluation and replication of miRNAs with disease stage and colorectal cancer-specific mortality. Int J Cancer 2015;137:428-438.

27 Shevde LA, Metge BJ, Mitra A, Xi Y, Ju J, King JA, Samant RS: Spheroid-forming subpopulation of breast cancer cells demonstrates vasculogenic mimicry via hsa-miR-299-5p regulated de novo expression of osteopontin. J Cell Mol Med 2010;14:1693-1706.

28 Huang M, Wu L, Luo S, Qin H, Yang Y, Chen J, Li Z, Qin Y: MicroRNA-1284 inhibits proliferation and induces apoptosis in SGC-7901 human gastric cancer cells. Biotechnol Lett 2017;39:33-38.

29 Song W, Li Q, Wang L, Wang L: Modulation of FoxO1 expression by miR-21 to promote growth of pancreatic ductal adenocarcinoma. Cell Physiol Biochem 2015;35:184-190.

-30 Cao J, Zhang Y, Yang J, He S, Li M, Yan S, Chen Y, Qu C, Xu L: NEAT1 regulates pancreatic cancer cell growth, invasion and migration though mircroRNA-335-5p/c-met axis. Am J Cancer Res 2016;6:2361-2374.

-31 Chen X, Wang L, Wang W, Zhao L, Shan B: B7-H4 facilitates proliferation of esophageal squamous cell carcinoma cells through promoting interleukin-6/signal transducer and activator of transcription 3 pathway activation. Cancer Sci 2016;107:944-954.

-32 Peng HX, Wu WQ Yang DM, Jing R, Li J, Zhou FL, Jin YF, Wang SY, Chu YM: Role of B7-H4 siRNA in Proliferation, Migration, and Invasion of LOVO Colorectal Carcinoma Cell Line. Biomed Res Int 2015;2015:326981.

-33 Xue Q Luan XY, Gu YZ, Wu HY, Zhang GB, Yu GH, Zhu HT, Wang M, Dong W, Geng YJ, Zhang XG: The negative co-signaling molecule b7-h4 is expressed by human bone marrow-derived mesenchymal stem cells and mediates its T-cell modulatory activity. Stem Cells Dev 2010;19:27-38.

34 Zhang J, Luo X, Li H, Deng L, Wang Y: Genome-wide uncovering of STAT3-mediated miRNA expression profiles in colorectal cancer cell lines. Biomed Res Int 2014;2014:187105.

-35 Hua W, Zhang M, Wang Y, Yu L, Zhao T, Qiu X, Wang L: Mechanical stretch regulates microRNA expression profile via NF- $\kappa$ B activation in C2C12 myoblasts. Mol Med Rep 2016;14:5084-5092.

-36 Prêtre V, Wicki A: Inhibition of Akt and other AGC kinases: A target for clinical cancer therapy? Semin Cancer Biol 2017.

-37 Dreas A, Mikulski M, Milik M, Fabritius CH, Brzózka K, Rzymski T: Mitogen-activated protein kinase (MAPK) interacting kinases 1 and 2 (MNK1 and MNK2) as targets for cancer therapy: recent progress in the development of MNK inhibitors. Curr Med Chem 2017;24:3025-3053.

-38 Zhang X, Cai L, Zhang G, Shen Y, Huang J: B7-H4 promotes tumor growth and metastatic progression in lung cancer by impacting cell proliferation and survival. Oncotarget 2017;8:18861-18871.

39 Wang X, Hao J, Metzger DL, Ao Z, Chen L, Ou D, Verchere CB, Mui A, Warnock GL: B7-H4 Treatment of T Cells Inhibits ERK, JNK, p38, and AKT Activation. PLoS One 2012;7:e28232. 